\title{
Neural systems that encode categorical versus coordinate spatial relations: PET investigations
}

\author{
STEPHEN M. KOSSLYN \\ Harvard University, Cambridge, Massachusetts \\ and Massachusetts General Hospital, Boston, Massachusetts \\ WILLIAM L. THOMPSON \\ Harvard University, Cambridge, Massachusetts \\ DARREN R. GITELMAN \\ Northwestern Medical School, Chicago, Illinois \\ and \\ NATHANIEL M. ALPERT \\ Massachusetts General Hospital, Boston, Massachusetts
}

\begin{abstract}
Participants received three sets of trials while regional cerebral blood flow was assessed using positron emission tomography (PET). In one set, the baseline, they responded when they detected a horizontal bar with an $X$ either above or below it. In another set, the categorical spatial relations judgment condition, they decided whether the $X$ was above or below the bar. In the third set, the coordinate spatial relations judgment condition, they decided whether the $\times$ was within 0.5 in. of the bar. In Experiment 1, the precise locations of the bars and $\times$ marks were varied, which required participants to focus attention selectively. Consistent with previous behavioral and neuropsychological findings, the left hemisphere was generally more activated during the categorical judgment task than during the coordinate judgment task, whereas the right hemisphere was generally more activated during the coordinate judgment task. In addition, these apparently simple tasks drew on large and different networks of areas. Experiment 2 was the same as Experiment 1 except that the stimuli were always in the same position and were presented very briefly. Three areas in the right parietal lobe were activated more by the coordinate task than by the categorical task in both experiments. In contrast, in neither experiment were any common areas activated more by the categorical task than by the coordinate task.
\end{abstract}

One way to formulate testable hypotheses about human information processing is to consider how one could build a machine that mimics specific human abilities. As a case in point, such considerations have suggested that our ability to encode spatial relations relies on at least two separate abilities (see Kosslyn, 1987, 1994). On the one hand, to reach to an object or navigate properly, metric spatial information must be represented relative to a point of origin. Such coordinate spatial relations representations presumably specify not only precise distance from an origin but also precise size and orientation (see Kosslyn, 1994). On the other hand, a robust description of the shape of a multipart, jointed object would rely on categories of spatial relations (e.g., above/below, inside/ outside, and on/off) that specify the type of spatial rela-

This research was supported by a grant from the Human Frontiers Science Program and by Grant N00014-94-1-0180 from the Office of Naval Research. The authors thank Steve Weise, Avis Loring, and the MGH Cyclotron Unit for technical assistance, and Christopher Chabris for helpful comments. Correspondence should be addressed to S. M. Kosslyn, 830 William James Hall, 33 Kirkland St., Cambridge, MA 02138 (e-mail: smk@wjh.harvard.edu). tions between parts. For example, if the person's shin is represented as "connected to" the thigh, this will describe all possible configurations of the two parts; thus, such categorical spatial relations representations can help one to identify an object even when it is contorted in novel ways, so that the precise shape varies from instance to instance. Many contemporary theories of object identification (e.g., Biederman, 1987; Marr, 1982) assume that objects are represented using "structural descriptions," which use categorical spatial representations to specify relations among parts. Indeed, Biederman's (1987) theory posits that coordinate spatial representations are not used in object identification, and Marr (1982) seemed to concur. The two types of representations are qualitatively distinct; what is critical for coordinate spatial relations representations is discarded when computing categorical representations.

Traditionally, the right cerebral hemisphere has been thought to be crucial for encoding and representing spatial relations (e.g., see Kolb \& Whishaw, 1990). However, the tasks that have typically been used to assess spatial encoding require one to encode metric information, and, hence, they would rely on coordinate spatial rela- 
tions representations (for a review, see Kosslyn, 1994). Kosslyn (1987) reasoned that the right hemisphere may be specialized for encoding coordinate spatial relations, whereas the left hemisphere's greater facility with language may generalize to a greater facility in forming categories, and, hence, categorical spatial relations might be encoded best in the left hemisphere. Three sets of converging evidence have supported the hypothesized hemispheric specialization.

First, findings from divided-visual-field experiments with normal people indicate that the hemispheres encode the two types of spatial relations differently. Kosslyn et al. (1989) found that when stimuli were presented in the left visual field (and, hence, initially encoded in the right hemisphere), participants could encode metric information faster than when stimuli were presented in the right visual field (and, hence, initially encoded in the left hemisphere). These results were obtained when participants judged whether a dot was within $1 \mathrm{~cm}$ of a blob, whether an $X$ was within 1 in. of an $O$, or whether a dot was within 0.5 in. of a horizontal bar. In contrast, when stimuli were presented in the right visual field (and, hence, encoded initially in the left hemisphere), participants could encode categorical information relatively faster than when the stimuli were presented in the left visual field. These results were obtained when participants determined whether a dot was on or off a blob, whether an $X$ was right or left of an $O$, and whether a dot was above or below a horizontal line. Hellige and Michimata (1989) devised the horizontal-bar-and-dot task and found the same results, and Koenig, Reiss, and Kosslyn (1990) found that even 5-year-olds exhibit these hemispheric differences. In addition, Laeng and Peters (1995) asked people to view objects and then to decide whether a lateralized version was identical. When objects were transformed in a categorical way to form the different trials, participants were faster to judge the target when it was presented in the right visual field (initially to the left hemisphere); in contrast, when the objects were transformed by altering their metric properties, participants were faster when stimuli were presented in the left visual field (initially to the right hemisphere). (However, although these results were replicated for right-handed participants, they were not found for left-handed females.) ${ }^{1}$

Second, results from studies of patients with unilateral brain damage also support the distinction between the two ways of encoding spatial relations. Laeng (1994) reports results that suggest that the posterior parietal lobe plays a special role in encoding each type of spatial relation representation. Such findings make sense because of the well-documented role of the posterior parietal lobes in encoding spatial information (e.g., see Andersen, 1987; Ungerleider \& Haxby, 1994; Ungerleider $\&$ Mishkin, 1982). Laeng found that patients with lefthemisphere damage had more difficulty noticing a change in categorical spatial relation than did patients with right-hemisphere damage, but he found the reverse pattern when the patients were asked to notice a change in a metric spatial relation. As expected, these results were pronounced for patients with damage only to the parietal lobes but also were present for patients who had damage to the temporal and frontal lobes (in conjunction with the parietal lobes). Laeng also obtained similar results when patients had to decide whether a previously studied object was presented in a reversed orientation (a categorical transformation) or with an altered extent on the horizontal or vertical axis (a metric transformation).

Finally, a series of computational models has shown that the two types of spatial relations are computed best under different circumstances. In particular, categorical spatial relations are computed best when the input is filtered through a set of small, nonoverlapping receptive fields, which make it easy to carve space into discrete "bins"; these small receptive fields can detect the presence or absence of two objects in specific relative positions. In contrast, coordinate spatial relations representations are computed best when the input is filtered through a set of large, overlapping receptive field, which promote "coarse coding"; the relative combined output from such input units allows the network to converge on the precise location of a stimulus (see Jacobs \& Kosslyn, 1994; Kosslyn, Chabris, Marsolek, \& Koenig, 1992, 1995). ${ }^{2}$ Cook, Früh, and Landis (1995) pointed out possible technical difficulties with these models, however, which led Baker, Chabris, and Kosslyn (in press) to construct new models that did not have these possible problems; the new models produced qualitatively the same results as the previous ones.

The difference in the ease of using outputs from neurons with large or small receptive fields to compute the two types of relations bears directly on findings concerning hemispheric specialization. Delis, Robertson, and Efron (1986), Robertson and Delis (1986), Sergent (1982), and others report that the left hemisphere is preferentially used when one encodes parts, whereas the right hemisphere plays a special role when one encodes global shapes. Thus, we expect the left hemisphere to be the major recipient of outputs from neurons with small receptive fields, and we expect the right hemisphere to be the major recipient of outputs from neurons with larger receptive fields. Kosslyn, Anderson, Hilger, and Hamilton (1994) tested this prediction by examining whether the hemispheres differ in the sizes of the pockets of space they encode easily. They asked participants to decide whether two successive line segments were presented at the same orientation. The distance between the segments was varied, but both members of the critical pairs were presented in the same visual field. In the first experiment, the participants evaluated segments that were relatively far apart more quickly when they were presented in the left visual field than when they were presented in the right visual field, which was expected if the right hemisphere does in fact preferentially receive input from units with larger receptive fields. Moreover, participants tended to judge segments that were relatively close together faster when they were pre- 
sented in the right visual field, which is consistent with the idea that the left hemisphere receives input from units with smaller receptive fields.

These results were replicated by Kosslyn, Anderson, et al. (1994) in a second experiment. However, they also found that the results changed dramatically when stimuli were moved farther toward the periphery, with participants now performing better for the widely separated segments when they were presented to the left hemisphere. These results suggested that the left hemisphere was set to encode information for the more difficult judgments. Similarly, the results were markedly different when participants had just performed the task with the stimuli presented at the other displacement toward the periphery (far or close). These results taken together suggested that the hemispheric differences observed in the first experiment were not due to hard-wired differences in outputs from neurons with different-sized receptive fields; rather, they were due to the way attention was allocated. That is, the hemispheres may in fact have neurons with the same distributions of receptive field sizes, but the outputs from neurons with larger receptive fields typically are processed preferentially in the right hemisphere, whereas those from neurons with smaller receptive fields typically are processed preferentially in the left hemisphere. However, this effect is due to attention selection, and it can be modified by task demands.

Part of our predictions, then, must rely on the inference that attentional processes play a key role in selecting outputs from neurons with different-sized receptive fields. These findings are particularly important for the present purposes because they imply that a network of brain areas is used to encode a particular type of spatial relation, if in fact one must set attention differently to encode the two types of relations. At present, there is only suggestive evidence that a network of brain areas is used to encode spatial relations. Kosslyn and Koenig (1995) note that results from a positron emission tomography (PET) study reported by Jonides et al. (1993) and by Smith and Jonides (1994) can be understood in part in terms of categorical versus coordinate encoding. In the spatial version of their task, participants had to remember the locations of three dots on a screen and then decide whether a small circle would have surrounded one of these dots. The precise metric locations of the dots relative to the sides of the screen had to be stored - a process that presumably relies on coordinate spatial relations. This task engendered activation, relative to a control condition, in four areas: right prefrontal cortex (Area 47, which may be analogous to Area 46 in the macaque; see Goldman-Rakic, 1987), the posterior portion of the right parietal cortex (Area 40), Area 19, and right premotor cortex (Area 6). Although this was a memory task (which may explain the role of dorsolateral prefrontal processing), the parietal lobes and Area 19 may play a role in setting attention and encoding spatial relations. The fact that these areas were activated on the right side is consistent with the need to encode coordinate representations of the locations.

In the present study, we sought to provide evidence that the two types of spatial relations are encoded using different mechanisms by demonstrating that different brain circuits are used to encode them; we expected networks of areas to be used to encode spatial relations but different networks to be used for categorical versus coordinate spatial relations. In sum, according to the present analysis, categorical spatial relations are encoded when the left hemisphere selects outputs from neurons with relatively small receptive fields, which allows it to carve space into small bins; the objects within these bins are identified, and the spatial relations between them are categorized. In contrast, coordinate spatial relations are encoded when the right hemisphere selects outputs from neurons with relatively large receptive fields, and coarsecoding mechanisms allow the specific metric locations of one or more points relative to an origin (often the point of focus) to be computed. In the present study, we directly compared categorical and coordinate spatial relations encoding while brain activation was monitored using PET.

\section{EXPERIMENT 1}

In the present experiments, we asked participants to view a horizontal bar with a single $X$ and to determine whether the $X$ was above or below the bar (a categorical judgment) or whether it was within 0.5 in. of the bar (a coordinate judgment). We compared activation in the two conditions not only with each other but also with a baseline condition in which participants saw the identical stimuli but simply responded when each one appeared. Identical stimuli were used in all three conditions; only the type of processing that was required was changed. We are led to make four predictions:

1. Given Laeng's (1994) results, we expect greater left parietal activation during the categorical task than during the coordinate one, but greater right parietal activation during the coordinate task than during the categorical one.

2 . The computer models suggested that the two types of spatial relations are computed best when one attends to outputs from units with different-sized receptive fields. The empirical findings indicated that such attention is actively allocated, not passively determined by hard-wired biases. If in fact the hemispheric differences in the relative ease of encoding the two types of spatial relations are due to attention, then we would expect activation of areas involved in visual attention during such tasks. There are many such areas, including the superior parietal lobe, inferior parietal lobe, pulvinar nucleus of the thalamus, superior colliculus, and anterior cingulate (e.g., see Corbetta, Miezen, Schulman, \& Petersen, 1993; Mesulam, 1981; Posner \& Petersen, 1990).

3. Coordinate judgments are performed primarily in the service of movement control. Indeed, it appears to be 
very rare that we use such information for anything other than guiding reaching or navigation. If so, then we might expect brain regions involved in planning movements, such as SMA, Area 6, the basal ganglia, or the cerebellum, to be activated when one encodes coordinate information. In contrast, there is no reason to expect these areas to be activated when one encodes categorical information.

4. Categorical spatial relations are specified in terms of one object relative to another. For example, a dot is above or below a bar. In order to make such a judgment, one must identify the objects. The same is not true for coordinate judgments of distance, which require only that one assess the relative distance between objects. Thus, we expect activation of inferior temporal regions in a categorical task, but not in a coordinate one; these regions have been shown to play a critical role in visual recognition (e.g., Fujita, Tanaka, Ito, \& Cheng, 1992; Haxby et al., 1991; Kosslyn, Alpert, et al., 1994; Levine, 1982; Sergent, Ohta, \& MacDonald, 1992; Sergent, Zuck, Lévesque, \& MacDonald, 1992; Ungerleider \& Haxby, 1994; Ungerleider \& Mishkin, 1982).

Perhaps the most basic prediction is that a large network of brain areas should be involved in computing the two types of spatial relations. Given the apparently simplicity of the tasks, this finding alone would be impressive.

\section{Method}

Participants. Eight right-handed males volunteered to take part as paid participants. All participants reported having normal or corrected-to-normal vision, and all reported being healthy and free of medication. The participants ranged in age from 22 years 3 months to 32 years 3 months, with a mean of 25 years 5 months. The participants were not aware of the experimental hypotheses until they were debriefed following the session. No one participated in more than one of the experiments reported in this article. We tested only males in this and the following experiment because of claims that males and females are lateralized differently; we worried that, by mixing the two, we would obtain a messy and inaccurate picture of either group.

Materials. All stimuli were created using the Aldus SuperPaint program, Version 3.5 for the Macintosh. First, an area 5 in. high $\times$ 2.5 in. wide was defined by four corner brackets; the brackets were formed by lines 0.1 in. long. Within the area defined by the brackets, there was a gray bar $l$ in. wide and 0.25 in. high. In addition, within the brackets was an $\times$ mark made up of two diagonal lines, each of which was approximately 0.25 in. long. Several versions of this stimulus were created. The gray bar appeared either just above the center of the area defined by the brackets or just below the center. The $X$ also could appear in different positions. On half the trials, the $X$ appeared above the gray bar; on half, it appeared below it. Moreover, on half of each of these types of trials, the $X$ appeared within 0.5 in. of the bar (a difficult categorical judgment); on the other half, it was more than 0.5 in. from the bar (an easy categorical judgment). The $X$ could also appear to the right or to the left of the center of the display, in one of four positions spaced equally along the horizontal extent of the bar. Finally, the $X$ could be close to the 0.5 -in. criterion distance from the bar-either just within or just outside this distance (a difficult coordinate judgment)-or could appear well within or outside the 0.5 -in. criterion (an easy coordinate judgment). Thus, there were six fully crossed conditions, each with two levels for a total of 64 different trials. (Bar above midline/below midline; $X$ above/below bar; $X$ inside/outside 0.5 in. from bar; $X$ in one of four positions along the bar [right/left and central/peripheral]; and judgment easy/difficult). These conditions were perfectly balanced so that each level of a condition was present on exactly half of the trials. No more than three trials in a row could have the same level of any condition. All stimuli appeared at the center of the screen.

Task procedure. The participants performed three tasks, with each administered in a separate block of trials. A PET scan was performed during each of these blocks. Identical stimuli, in the identical order, were presented in all three tasks; only the instructions given to the participants varied from one block of trials to the next. For all three tasks, the structure of the trials proceeded as follows: First, a blank screen appeared for $1,000 \mathrm{msec}$, followed by the stimulus (bar and $X$ ). The stimulus remained on the screen until the participant responded by pressing a foot pedal. At that point, the blank screen returned, and a new trial began.

In the baseline condition, the participants were instructed simply to view the stimuli that appeared on the screen and to press one of the pedals under their feet as soon as they saw each stimulus. The participants were asked to alternate the foot of response over trials, so that, if they began with the left foot, they would next respond with the right, then the left, and so on; half the participants were instructed to begin responding with the left foot, and half were instructed to begin with the right. The participants read instructions on the screen before beginning the task, after which the investigator asked them to paraphrase the instructions. Any misconceptions were corrected, and the participants were encouraged to ask for clarification if needed. All participants received the baseline task first, because we were concerned that, if it followed the other conditions, they would spontaneously perform one of the other tasks. Across subjects, the remaining two conditions were presented equally often in each order (categorical-coordinate, coordinatecategorical).

In the categorical condition, the participants were asked to decide, as quickly and accurately as possible, whether the $X$ mark appeared above or below the gray bar. When it appeared above the bar, they were to press one pedal; when it appeared below the bar, they were to press the other pedal. The number of participants using each pedal to signal a given judgment was counterbalanced within each group (defined by the order in which the conditions were administered). In all other respects, the procedure was the same as for the baseline task.

In the coordinate condition, the participants were asked to decide whether the $X$ was within $0.5 \mathrm{in}$. of the bar (inside) or more than 0.5 in. from the bar (outside). When the $X$ appeared within 0.5 in. of the bar, the participants were to press one pedal; when the $X$ was farther than 0.5 in. from the bar, they were to press the other. The pedal of response for each response type (inside and outside) was counterbalanced so that an equal number of participants responded with each pedal for each of the two response types, within each of the two order groups. In all other respects, the procedure was the same as for the baseline task.

In both the categorical and the coordinate condition, the participants were shown examples of each type of trial $(X$ above bar vs. $X$ below bar, or $X$ inside 0.5 in. vs. $X$ outside 0.5 in.) on the screen prior to the test trials. For the coordinate task, they were shown what a distance of $0.5 \mathrm{in}$. looked like on the screen, using an illustration of a gray bar and an $X$ like those in the test trials. The participants were asked to remember what $0.5 \mathrm{in}$. looked like on the screen, and they were shown two examples of inside trials and two examples of outside trials, with a dashed line to indicate the 0.5 -in. mark. The participants had a clear idea of the extent of 0.5 in., and this demonstration was simply to let them know what that distance looked like in this situation; no participant reported having difficulty understanding this criterion.

PET procedure. In each condition, after it was clear that the participants understood the nature of the task, the investigator waited for the signal from the PET technologist that the PET camera had 
been turned on. Fifteen seconds after this signal, the participant was asked to begin the task; $15 \mathrm{sec}$ later $\mathrm{O}^{15}$ began to be delivered and continued for $1 \mathrm{~min}$. After the flow of $\mathrm{O}^{15}$ stopped, the participants continued the task for approximately $15 \mathrm{sec}$, at which point the PET camera was turned off. The $\mathrm{O}^{15}$ was allowed to decay for $10 \mathrm{~min}$ before the next condition. During this time, the participants received the instructions for the following task. In all other respects, the PET procedure was identical to that used in Kosslyn, Alpert, et al. (1994).

\section{Results}

We performed separate analyses of the behavioral and PET results.

Behavioral analyses. The participants required more time to perform the coordinate task (with means of 721 and $531 \mathrm{msec}$ for coordinate and categorical judgments, respectively) $[F(1,7)=100.24, p=.0001]$. They also required more time to evaluate the more difficult trials (with means of 644 and $607 \mathrm{msec}$ for difficult and easy trials, respectively) $[F(1,7)=10.32, p<.05]$. However, the difference in difficulty occurred only in the coordinate trials. There was an interaction between task and difficulty (with means of 526 and $535 \mathrm{msec}$ for the difficult and easy trials in the categorical task, and 762 and $680 \mathrm{msec}$ for the difficult and easy trials in the coordinate task) $[F(1,7)=19.29, p<.005]$. Contrasts also revealed no difference between the difficult and easy conditions of the categorical task $[t(7)<1]$ but did reveal a difference between the difficult and easy conditions of the coordinate task $[t(7)=5.63, p<.001]$. The participants made comparable numbers of errors during the two tasks (with means of $3.6 \%$ and $6.0 \%$ errors for the categorical and coordinate tasks, respectively) $[F(1,7)=$ $1.42, p>.4]$. No other comparisons of error rates, including task order, were significant $(p>.1)$.
The longer response times in the coordinate task resulted in the participants' receiving a mean of 54.1 trials in this condition relative to 60.9 trials in the categorical condition, but this difference was not significant $[F(1,7)=$ $1.14, p>.3]$. We also computed the total amount of time the participants spent processing each task during each condition, simply by summing the times from the presentation of each stimulus to the response. The participants spent $32.1 \mathrm{sec}$ processing categorical judgments and $38.9 \mathrm{sec}$ processing coordinate judgments $[t(7)=$ $2.32, p=.03]$.

PET analyses. We performed contrasts to compare relative cerebral blood flow in the different conditions. These analyses made use of the SPM (statistical parametric mapping) program (Friston, Frith, Liddle, \& Frackowiak, 1991). The method of analysis was identical to that described by Kosslyn, Alpert, et al. (1994).

Categorical versus baseline. We began by comparing each of the test conditions with the baseline. The results of contrasting blood flow in the categorical condition with that in the baseline are presented in Table 1 and Figure 1.

Consider our predictions. As predicted, we found activation in the left inferior parietal lobe, which we expected if this structure plays a key role either in encoding categorical spatial relations themselves (as suggested by Laeng, 1994) or in setting attention properly for this type of judgment. We also found activation in the left Area 19, which may play a role in the categorization process itself.

In addition, we expected activation of areas involved in visual attention. We found activation bilaterally in part of the precentral gyrus that may correspond to the frontal eye fields (Paus, 1996). Moreover, we found activation

Table 1

Blood Flow in the Categorical Spatial Relations Encoding Task Compared With the Baseline Task in Experiment 1, in Which Stimuli Were Presented in Free View

\begin{tabular}{|c|c|c|c|c|c|c|}
\hline Area & $X$ & $Y$ & $Z$ & $Z$ Score & $\begin{array}{c}p \\
\text { (hyp) }\end{array}$ & $\begin{array}{c}p \\
\text { (no hyp) }\end{array}$ \\
\hline \multicolumn{7}{|c|}{ Left-Hemisphere Regions } \\
\hline Area 19 & -12 & -36 & 0 & 4.03 & .0006 & .02 \\
\hline Inferior parietal & -54 & -34 & 48 & 3.49 & .007 & .06 \\
\hline Medial frontal & -10 & -10 & 52 & 3.81 & .002 & .02 \\
\hline Precentral gyrus & -38 & -7 & 56 & 3.45 & .004 & .05 \\
\hline \multicolumn{7}{|c|}{ Right-Hemisphere Regions } \\
\hline Superior parietal & 25 & -55 & 56 & 3.78 & .0008 & .02 \\
\hline Middle temporal & 41 & -54 & 12 & 3.73 & .001 & .05 \\
\hline Inferior parietal & 45 & -49 & 48 & 3.13 & .02 & \\
\hline Postcentral gyrus & 18 & -41 & 64 & 3.71 & .002 & .006 \\
\hline Precentral gyrus & 40 & -21 & 52 & 3.92 & .0006 & .01 \\
\hline Anterior cingulate & 10 & 30 & 24 & 4.16 & .0001 & .008 \\
\hline \multicolumn{7}{|c|}{ Midline Regions } \\
\hline Superior colliculus & l & -34 & -4 & 4.11 & .00004 & .01 \\
\hline
\end{tabular}

Note-Coordinates are relative to the anterior commissure. $X$ is the horizontal dimension (with positive toward the right); $Y$ is the depth dimension (with positive toward the anterior part of the brain); $Z$ is the vertical dimension (with positive toward the top). The last two columns of the table indicate the probability $(p)$ levels associated with the listed region, depending on the presence (hyp) or absence (no hyp) of a hypothesis for that region. 


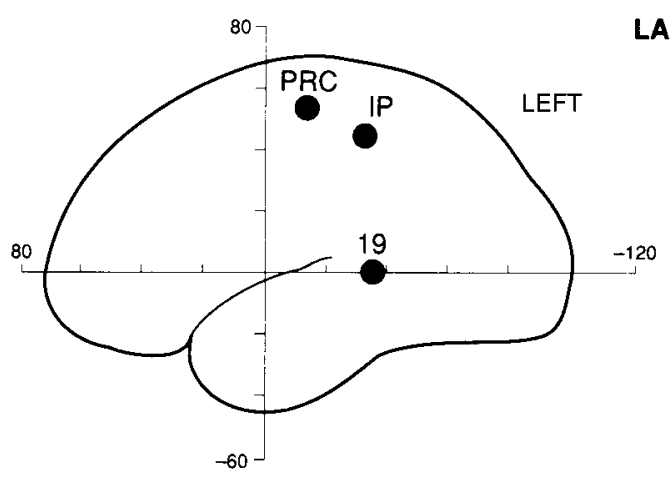

LATERAL VIEW
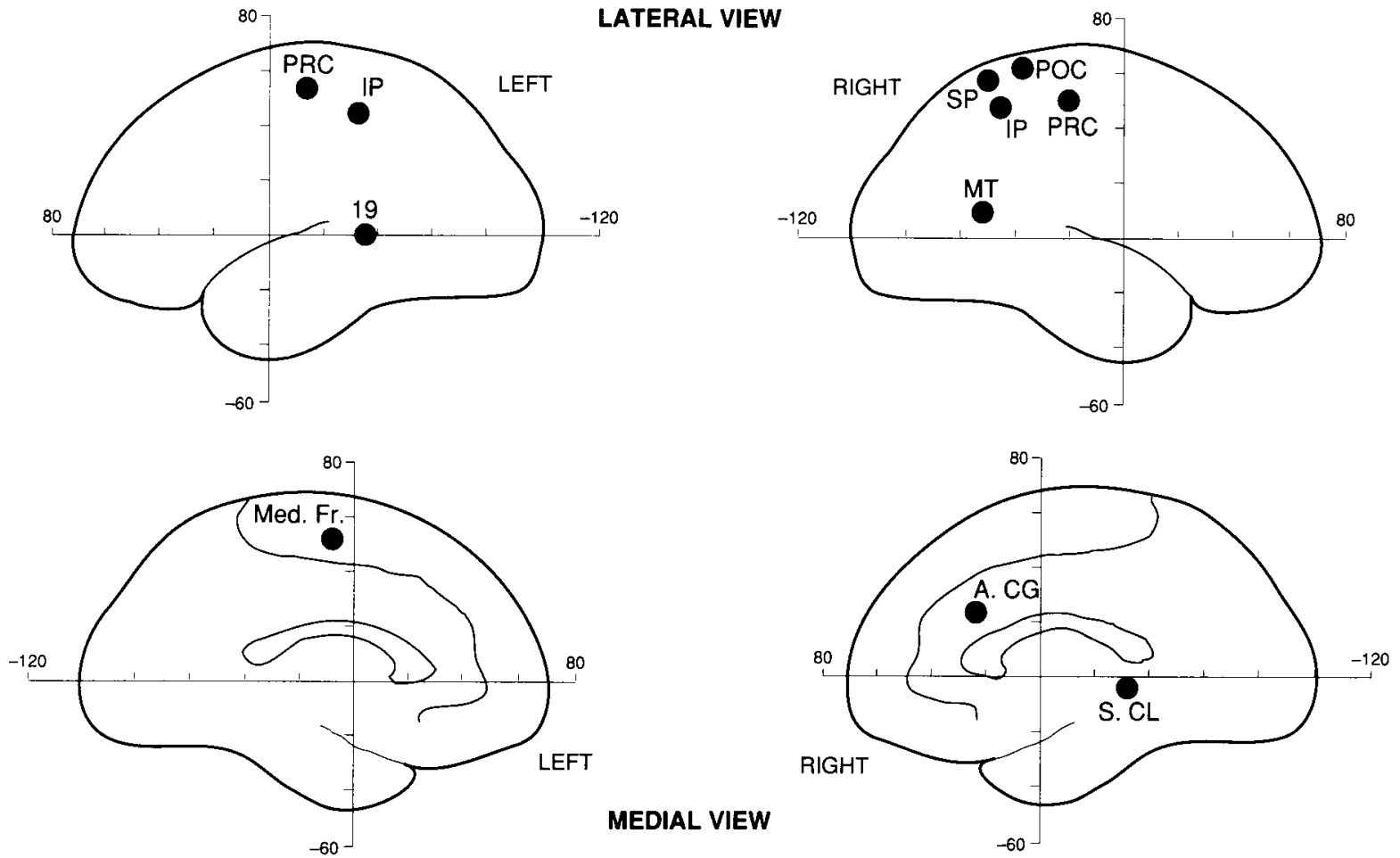

Figure 1. Areas in which there was significantly greater blood flow in the categorical spatial relations encoding condition than in the baseline condition in Experiment 1. Left hemisphere is on the left; right hemisphere is on the right. Points on the lateral surface are at the top; points on the medial surface are at the bottom. Points indicate the location of the most significant pixel in an area. The axes indicate location in $\mathbf{2 0}-\mathbf{m m}$ increments relative to the anterior commissure.

in the right superior parietal lobe, right inferior parietal lobe, right anterior cingulate, and superior colliculus, all of which are likely to play roles in visual attention (e.g., Posner \& Petersen, 1990).

We also predicted activation of inferior temporal regions in a categorical task, but not in a coordinate one, and such activation was in fact evident but in the right hemisphere.

Finally, activation was evident in the left superior frontal area and right postcentral gyrus, neither of which was expected.

Coordinate versus baseline. Table 2 and Figure 2 present the results of contrasting the coordinate task with the baseline. Two general results are immediately evident: First, the pattern is very different from that in Table 1 and Figure 1, which provides good evidence that different brain systems are at work when the two types of spatial relations are encoded. Second, there is a large network of areas, as expected if the underlying processing is as complicated as our analysis suggests.

Regarding our specific predictions, we found activation of the right superior parietal lobe (but not inferior parietal cortex). We again expected activation of areas involved in visual attention, and we found bilateral activation of the thalamus (the pulvinar on the left, dorsomedial on the right), bilateral Area 8 (the supplementary eye fields), bilateral superior parietal cortex, precuneus, and superior colliculus. All of these areas have been taken to play roles in attention (e.g., see Corbetta et al., 1993; Posner \& Petersen, 1990). In addition, we found activation of the left caudate and right putamen, which are part of the striatum; these structures receive motor input from the cortex, transform it, then send it back to the cortex via the thalamus. We also found activation that was centered in the precentral gyrus, but this activation clearly extended into Area 6 . Finally, we found activation in the right dorsolateral prefrontal area (the center of the activation is located in Area 9, but there is some extension into Area 46 on the slice immediately inferior, Slice 24 ; the point at coordinates $50,24,24$ reaches a $Z$ score of 3.78 and is located within Area 46) and right superior frontal cortex, in an area right on the border of Area 8, the supplementary eye fields.

Comparing test conditions. Finally, Table 3 and Figure 3 present the results of directly comparing the two test conditions. As is evident, the only areas that were more active in the categorical task than in the coordinate task were the left dorsolateral prefrontal area and the left superior temporal area. The former region is classified as Area 46 by the Talairach and Tournoux (1988) atlas, which is similar to that identified by Jonides et al. (1993). The 
Table 2

Blood Flow in the Coordinate Spatial Relations Encoding Task Compared With the Baseline Task in Experiment 1, in Which Stimuli Were Presented in Free View

\begin{tabular}{|c|c|c|c|c|c|c|}
\hline Area & $X$ & $Y$ & $Z$ & $Z$ Score & $\begin{array}{c}p \\
\text { (hyp) }\end{array}$ & $\begin{array}{c}p \\
\text { (no hyp) } \\
\end{array}$ \\
\hline \multicolumn{7}{|c|}{ Left-Hemisphere Regions } \\
\hline Superior parietal & -34 & -69 & 56 & 4.32 & .0001 & .002 \\
\hline Area 19 (superior) & -30 & -62 & 36 & 3.66 & .002 & .05 \\
\hline Thalamus (pulvinar) & -20 & -35 & 4 & 4.99 & .000006 & .002 \\
\hline DLPFC & -44 & -16 & 52 & 4.17 & .0005 & .05 \\
\hline Caudate & -11 & 21 & 8 & 4.20 & .00007 & .008 \\
\hline Area 8 & -20 & 39 & 48 & 4.00 & .0008 & .01 \\
\hline \multicolumn{7}{|c|}{ Right-Hemisphere Regions } \\
\hline Area 18 & 15 & -78 & 28 & 4.26 & .00004 & .006 \\
\hline Inferior parietal & 38 & -71 & 48 & 4.08 & .0008 & .008 \\
\hline Superior parietal & 18 & -48 & 64 & 3.94 & .0008 & .006 \\
\hline Precentral gyrus & 25 & -26 & 68 & 4.75 & .00003 & .0001 \\
\hline Putamen & 24 & -18 & 16 & 4.55 & .00002 & .002 \\
\hline Thalamus (dorsomedial) & 6 & -16 & 12 & 3.78 & .0008 & .04 \\
\hline Inferior frontal & 35 & 16 & 12 & 4.75 & .0002 & .003 \\
\hline DLPFC (Area 9) & 50 & 19 & 28 & 5.17 & $<.00001$ & $<.001$ \\
\hline Superior frontal & 10 & 38 & 32 & 3.93 & .0009 & .02 \\
\hline \multicolumn{7}{|c|}{ Midline Regions } \\
\hline Precuneus & 4 & -59 & 60 & 3.90 & .0005 & .01 \\
\hline Superior colliculus & 1 & -40 & -4 & 4.72 & .000003 & .0008 \\
\hline Medial frontal & 1 & 25 & 60 & 4.26 & .0002 & .002 \\
\hline
\end{tabular}

Note-Coordinates are relative to the anterior commissure. $X$ is the horizontal dimension (with positive toward the right); $Y$ is the depth dimension (with positive toward the anterior part of the brain); $Z$ is the vertical dimension (with positive toward the top). The last two columns of the table indicate the probability $(p)$ levels associated with the listed region, depending on the presence (hyp) or absence (no hyp) of a hypothesis for that region. DLPFC indicates dorsolateral prefrontal cortex.

reverse comparison revealed that the left inferior parietal area and left Area 19 were more active in the coordinate task than in the categorical one; in addition, in the right hemisphere, the precuneus, superior parietal lobe, inferior frontal lobe, and dorsolateral prefrontal (Area 46) were activated more in the coordinate task.

We were concerned that the observed differences might simply reflect the fact that the coordinate task was more difficult. Thus, we lowered the significance threshold for the categorical task to the point where an equal number of active areas were evident in the two tasks. Even here, different areas were activated in the two tasks: In addition to the superior temporal gyrus and left dorsolateral prefrontal cortex, there was subthreshold activation of the left anterior cingulate, postcentral gyrus, supplementary eye fields, and middle temporal gyrus during the categorical task.

Thus, we found more areas activated in the left hemisphere than in the right hemisphere during the categorical task, but more areas activated in the right hemisphere than in the left during the coordinate task. These results line up with the observed response time differences in divided-visual-field studies, but they do not explain the apparent critical role of the posterior parietal lobe posited by Laeng (1994). The only homologous area that was asymmetrically activated by the two tasks was Area 46, in the frontal lobes.

\section{Discussion}

We found activation in many areas that are involved in attention, as expected if one must adjust attention appropriately to encompass the $X$ and bar. Moreover, we found left-hemisphere activation during the categorical task and predominantly right-hemisphere activation during the coordinate task (when the two were directly compared). In addition, inferior temporal regions were activated in the categorical task but not in the coordinate task, and motor areas were activated in the coordinate task but not in the categorical one, as predicted. Perhaps our most basic prediction was that a large network of brain areas would be involved in computing the two types of spatial relations, many of which would be involved in the attentional processes needed to select outputs from neurons with different-sized receptive fields. This was indeed the case.

However, when we directly compared the categorical and coordinate conditions, we did not find relatively more activation in the left parietal lobe during the categorical task. Nevertheless, we found that the coordinate task evoked greater activation than did the categorical task in a portion of the right superior parietal lobe and a portion of the right precuneus, which is within the medial superior parietal lobe; it is possible that these two regions play a key role in the computation of metric spa- 

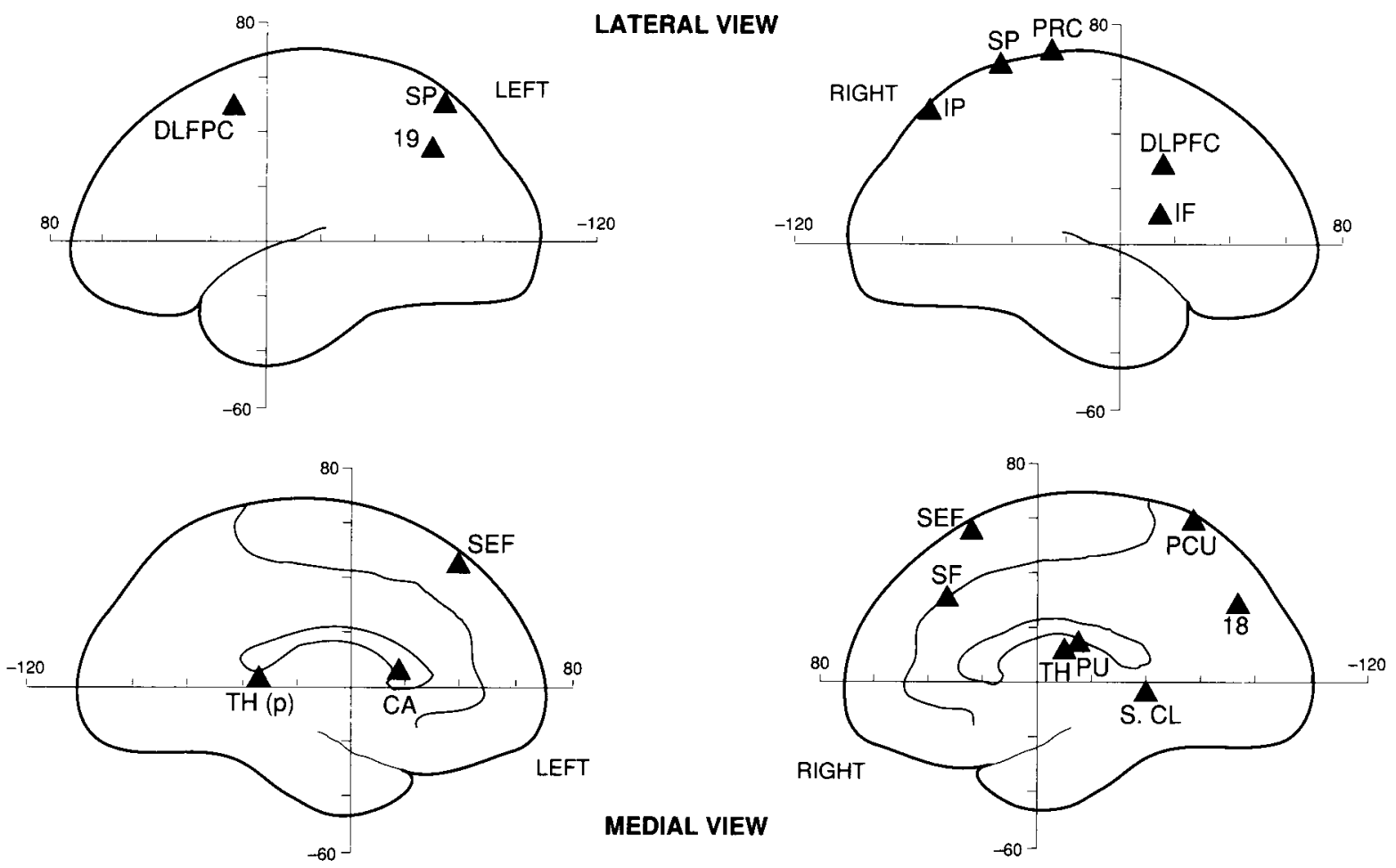

Figure 2. Areas in whicb there was significantly greater blood flow in the coordinate spatial relations encoding condition than in the baseline condition in Experiment 1. Left hemisphere is on the left; right hemisphere is on the right. Points on the lateral surface are at the top; points on the medial surface are at the bottom. Points indicate the location of the most significant pixel in an area. The axes indicate location in $20-\mathbf{m m}$ increments relative to the anterior commissure.

Table 3

Blood Flow in the Categorical Spatial Relations Encoding Task Compared With the Coordinate Task in Experiment 1, in Which Stimuli Were Presented in Free View

\begin{tabular}{ccccccc}
\hline Area & $X$ & $Y$ & $Z$ & $Z$ Score & $\begin{array}{c}p \\
\text { (hyp) }\end{array}$ & $\begin{array}{c}p \\
\text { (no hyp) }\end{array}$ \\
\hline
\end{tabular}

Categorical-Coordinate

Superior temporal

Left-Hemisphere Regions

DLPFC (Area 46)

$\begin{array}{rrrrrr}-43 & -21 & -4 & 3.72 & .001 & .05 \\ -29 & 38 & 24 & 3.76 & .002 & .04\end{array}$

Coordinate-Categorical

Left-Hemisphere Regions

Area 19

Inferior parietal

$\begin{array}{llllll}-34 & -77 & 16 & 4.15 & .0005 & .01 \\ -34 & -64 & 36 & 3.53 & .005 & .08\end{array}$

Precuneus

Superior parietal

Inferior frontal

Right-Hemisphere Regions

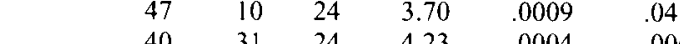

Note-Coordinates are relative to the anterior commissure. $X$ is the horizontal dimension (with positive toward the right); $Y$ is the depth dimension (with positive toward the anterior part of the brain); $Z$ is the vertical dimension (with positive toward the top). The last two columns of the table indicate the probability $(p)$ levels associated with the listed region, depending on the presence (hyp) or absence (no hyp) of a hypothesis for that region. DLPFC indicates dorsolateral prefrontal cortex. 

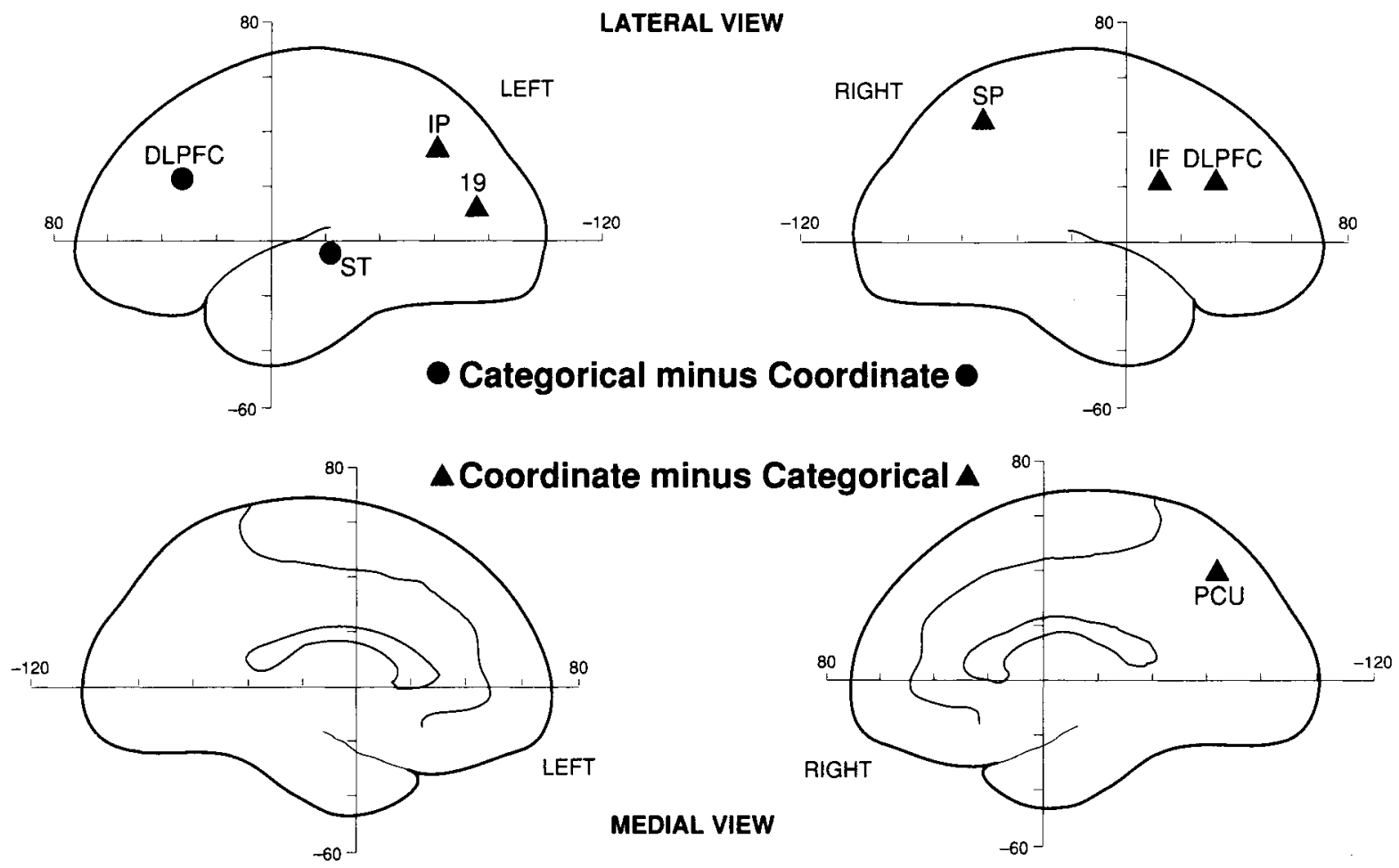

Figure 3. Areas in which there was significantly greater blood flow in the categorical spatial relations encoding condition than in the coordinate spatial relations encoding condition (circles), or vice versa (triangles), in Experiment 1. Left hemisphere is on the left; right hemisphere is on the right. Points on the lateral surface are at the top; points on the medial surface are at the bottom. Points indicate the location of the most significant pixel in an area. The axes indicate location in $20-\mathrm{mm}$ increments relative to the anterior commissure.

tial information. Given the sheer number of brain areas that were activated, it is possible that differences in the activation of small areas were masked by larger differences in surrounding areas.

\section{EXPERIMENT 2}

Our interpretation of the findings of Experiment 1 rests on the notion that the participants actively allocated attention to facilitate making the different judgments. Experiment 1 was designed to maximize the contribution of these processes by requiring the participants to find the bar and then the $X$ prior to making the judgment. However, none of the previous experiments in the literature varied the stimuli in this way, which may be why we did not find the posterior parietal lobe activated more on the left side when categorical spatial relations were encoded.

Accordingly, we modified the stimuli of Experiment 1 to reduce the role of search processes during attention. We did this by changing three aspects of the stimuli. First, the bar always appeared in same location on the screen. Second, the $X$ appeared only over or under the middle of the bar. Third, the stimuli remained visible only for $200 \mathrm{msec}$, not enough time to make eye movements. All three of these manipulations were expected to minimize the use of processes that shift attention to specific regions of space and make the experiment more similar to those reported in the past.

\section{Method}

Participants. Eight right-handed males volunteered to take part as paid participants. All participants reported having normal or corrected-to-normal vision, and all reported being healthy and free of medication. The participants ranged in age from 18 years 5 months to 34 years 5 months, with a mean of 22 years 9 months. The participants were not aware of the experimental hypotheses until they were debriefed following the session.

Materials. The materials were identical to those used in Experiment 1 except that (1) the bar always appeared at the midline of the field defined by the brackets and (2) the $X$ always appeared centered above or below the horizontal bar. The conditions were perfectly balanced so that each level of a condition was present on exactly half of the trials. No more than three trials in a row could have the same level of any condition. All stimuli appeared at the center of the screen.

Task and PET procedure. The procedure was identical to that of Experiment 1 except that the stimuli were exposed for only $200 \mathrm{msec}$ and a fixation point appeared at the beginning of each trial instead of a blank screen.

\section{Results}

We again performed separate analyses of the behavioral and PET results.

Behavioral analyses. The participants required more time for the coordinate task than for the categorical task 
(with means of 550 and $365 \mathrm{msec}$, respectively) $[F(1,7)=$ $37.99, p<.0001]$. The participants also required more time to make more difficult discriminations (500 and $414 \mathrm{msec})[F(1,7)=28.73, p=.001]$. The interaction between task and difficulty $[F(1,6)=19.65, p=.003]$ indicated that the difference in difficulty was restricted to the coordinate task. Contrasts revealed an effect of difficulty in the coordinate task $[t(7)=6.6, p=.0003]$, but not in the categorical task $[t(7)=.33, p>.7]$.

In addition, analyses of errors revealed no difference between the tasks $[F(1,7)=2.45, p>.16]$ but did show that the participants made more errors for difficult conditions $(6.9 \%)$ than for easy ones $(3.0 \%)$; however, an interaction of task and difficulty revealed that the participants' error rates were $3.8 \%$ and $2.7 \%$, respectively, for the difficult and easy conditions of the categorical task and were $10.0 \%$ and $3.1 \%$, respectively, for the difficult and easy conditions of the coordinate task $[F(1,7)=$ $7.47, p<.03]$. Contrasts revealed an effect of difficulty in the coordinate task $[t(7)=4.5, p<.003]$, but not the categorical task $[t(7)=.64, p>.5]$.

The longer response times in the coordinate task resulted in the participants' receiving a mean of 62.4 trials in this condition relative to 67.3 trials in the categorical condition, but this difference was not significant $[F(1,7)=$ $1.79, p>.2]$. We again computed the total amount of time spent processing the stimuli in the two conditions and again found that the participants spent more time processing coordinate judgments $(34.2 \mathrm{sec})$ than they did processing categorical judgments $(23.4 \mathrm{sec})[t(7)=$ $4.16, p=.001]$.

PET analyses. The PET data were analyzed as in Experiment 1.

Categorical versus baseline. As before, we began by comparing each of the test conditions with the baseline. The results of contrasting blood flow in the categorical condition with that in the baseline are presented in Table 4 and Figure 4.
Consider our predictions. As is evident, we did find activation in the left inferior parietal lobe. In addition, we found evidence that attention plays a role in this sort of processing, even when the participant does not need to search for the stimuli; we found activation in the right superior parietal lobe, which is likely to play a key role in shifting attention (e.g., Corbetta et al., 1993). However, unlike in Experiment 1, activation of inferior temporal regions was not evident.

In addition, we found activation in Area 19, but it was on the right side. We also found unexpected activation of the left dorsolateral prefrontal area, but it was within Area 9, and also of the right angular gyrus.

Coordinate versus baseline. Table 5 and Figure 5 present the results of contrasting the coordinate task with the baseline. As is evident, we found activation in the right superior parietal lobe. In addition, we found activation in several areas that play roles in attentionspecifically, the right thalamus and the left cingulate gyrus (e.g., see Posner \& Petersen, 1990). We did not, however, find activation in motor cortex. However, we did find activation in the right dorsolateral prefrontal area (part of Area 6), and we were surprised to find bilateral activation of the superior frontal areas and, particularly, of a region identified as Broca's area.

Comparing test conditions. Finally, Table 6 and Figure 6 present the results of directly comparing the two test conditions. As is evident, the only area that was more activated in the categorical task than in the coordinate task was in the left frontal lobe. In addition, the reverse comparison revealed that the left inferior and right superior parietal areas were more active in the coordinate task than in the categorical one.

\section{Discussion}

Fewer areas were activated when the stimuli were presented in the same location on the screen and for less time than one needs to make an eye movement. As expected,

Table 4

Blood Flow in the Categorical Spatial Relations Encoding Task Compared With the Baseline Task in Experiment 2, in Which Stimuli Were Presented for 200 msec and Were Aligned Along the Midline

\begin{tabular}{|c|c|c|c|c|c|c|}
\hline Area & $X$ & $Y$ & $Z$ & Z Score & $\begin{array}{c}p \\
\text { (hyp) }\end{array}$ & $\begin{array}{c}p \\
\text { (no hyp) }\end{array}$ \\
\hline \multicolumn{7}{|c|}{ Left-Hemisphere Regions } \\
\hline DLPFC & -55 & 11 & 36 & 3.48 & .006 & \\
\hline Superior frontal (supplementary eye fields) & -16 & 24 & 52 & 3.59 & .005 & .04 \\
\hline Area 19 & 34 & -83 & 20 & 3.81 & .001 & .04 \\
\hline Area 18 & 36 & -78 & 12 & 4.46 & .00005 & .003 \\
\hline Angular gyrus & 26 & -73 & 28 & 3.95 & .001 & .02 \\
\hline Superior parietal & 21 & -53 & 44 & 3.69 & .001 & .04 \\
\hline
\end{tabular}

Note - Coordinates are relative to the anterior commissure. $X$ is the horizontal dimension (with positive toward the right); $Y$ is the depth dimension (with positive toward the anterior part of the brain); $Z$ is the vertical dimension (with positive toward the top). The last two columns of the table indicate the probability $(p)$ levels associated with the listed region, depending on the presence (hyp) or absence (no hyp) of a hypothesis for that region. DLPFC indicates dorsolateral prefrontal cortex. 


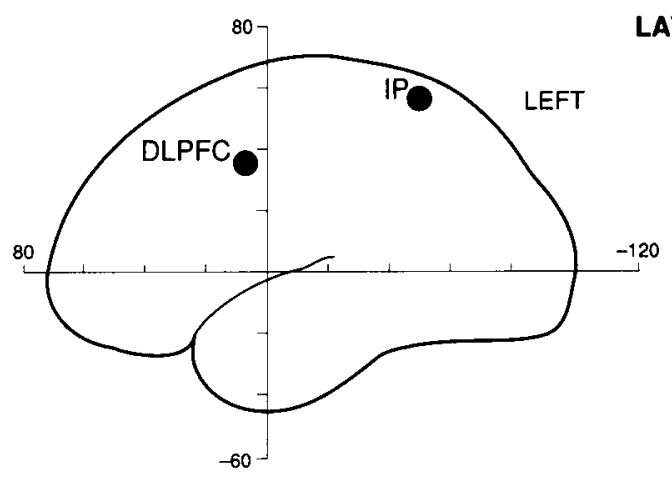

LATERAL VIEW
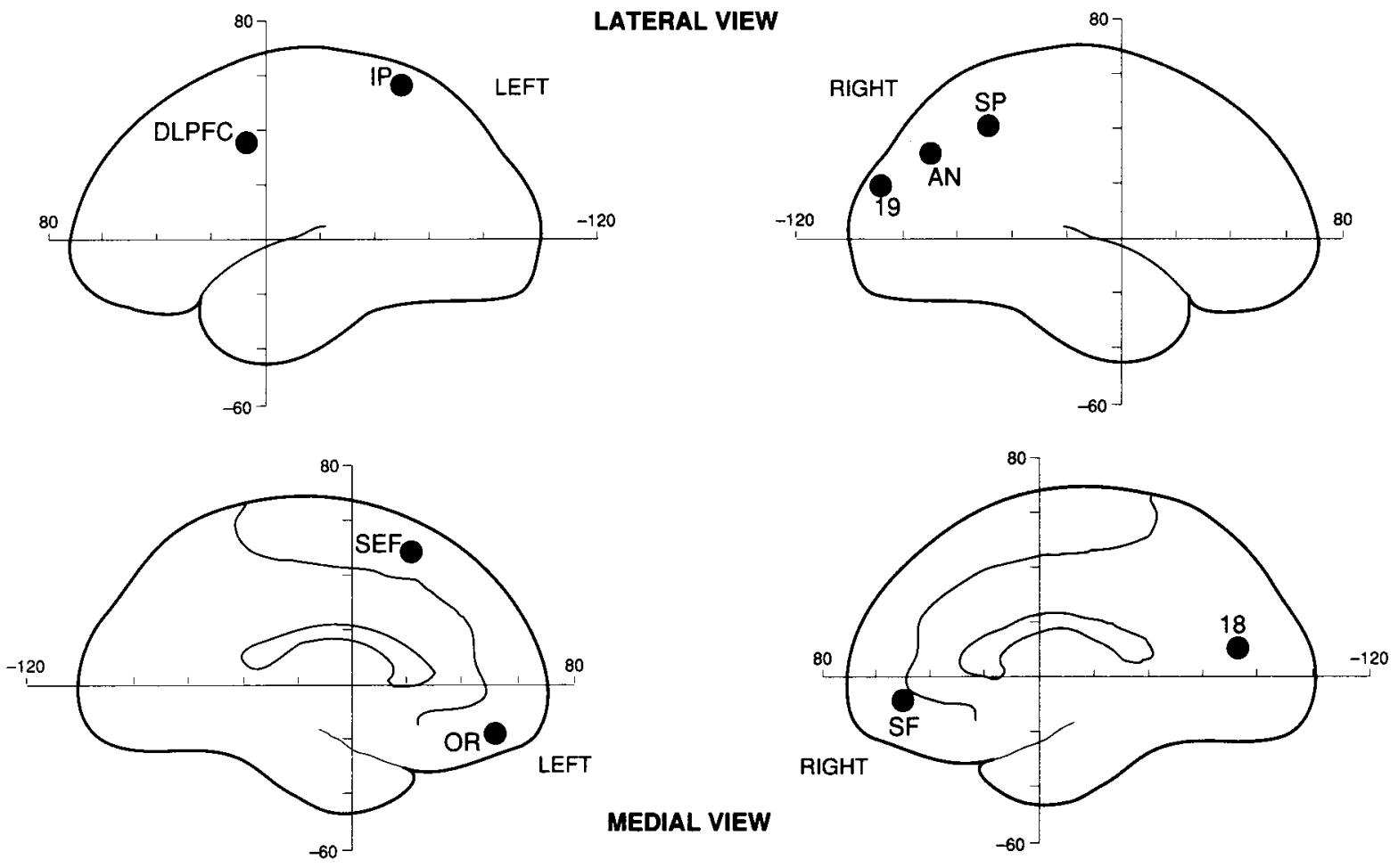

Figure 4. Areas in which there was significantly greater blood flow in the categorical spatial relations encoding condition than in the baseline condition in Experiment 2 . Left hemisphere is on the left; right hemisphere is on the right. Points on the lateral surface are at the top; points on the medial surface are at the bottom. Points indicate the location of the most significant pixel in an area. The axes indicate location in $20-\mathrm{mm}$ increments relative to the anterior commissure.

many of the areas that were no longer present are involved in visual search, and some - such as the frontal eye fields - are involved in making eye movements per se. Indeed, the lack of activation in the inferior temporal cortex in this situation may suggest that the participants did need to recognize the bar in Experiment 1, whereas they did not need to do so here. In addition, we expected a subset of the areas activated in Experiment 1 to be activated in Experiment 2. When we compare the locations of the most-activated pixels in the two experiments, a

Table 5

Blood Flow in the Coordinate Spatial Relations Encoding Task Compared With the Baseline Task in Experiment 2, in Which Stimuli Were Presented for 200 msec and Were Aligned Along the Midline

\begin{tabular}{|c|c|c|c|c|c|c|}
\hline Area & $X$ & $Y$ & $Z$ & $Z$ Score & $\begin{array}{c}p \\
\text { (hyp) }\end{array}$ & $\begin{array}{c}p \\
\text { (no hyp) }\end{array}$ \\
\hline \multicolumn{7}{|c|}{ Left-Hemisphere Regions } \\
\hline Inferior parietal & -58 & -31 & 48 & 2.89 & .04 & \\
\hline Medial frontal & -10 & 6 & 44 & 4.08 & .0007 & .008 \\
\hline Cingulate & -10 & 17 & 28 & 4.31 & .0002 & .005 \\
\hline Broca's area (Area 45) & -45 & 35 & 8 & 3.71 & .003 & .05 \\
\hline \multicolumn{7}{|c|}{ Right-Hemisphere Regions } \\
\hline Superior parietal & 27 & -59 & 44 & 3.25 & .006 & \\
\hline Insula & 33 & -26 & 4 & 3.70 & .001 & .05 \\
\hline Thalamus (nucleus accumbens) & 6 & -7 & 16 & 4.15 & .0001 & .01 \\
\hline DLPFC & 24 & -3 & 60 & 3.23 & .01 & \\
\hline Prefrontal area & 20 & 51 & -12 & 3.19 & .03 & \\
\hline Superior frontal & 13 & 66 & 4 & 3.34 & .004 & \\
\hline
\end{tabular}

Note-Coordinates are relative to the anterior commissure. $X$ is the horizontal dimension (with positive toward the right); $Y$ is the depth dimension (with positive toward the anterior part of the brain); $Z$ is the vertical dimension (with positive toward the top). The last two columns of the table indicate the probability $(p)$ levels associated with the listed region, depending on the presence (hyp) or absence (no hyp) of a hypothesis for that region. DLPFC indicates dorsolateral prefrontal cortex. 

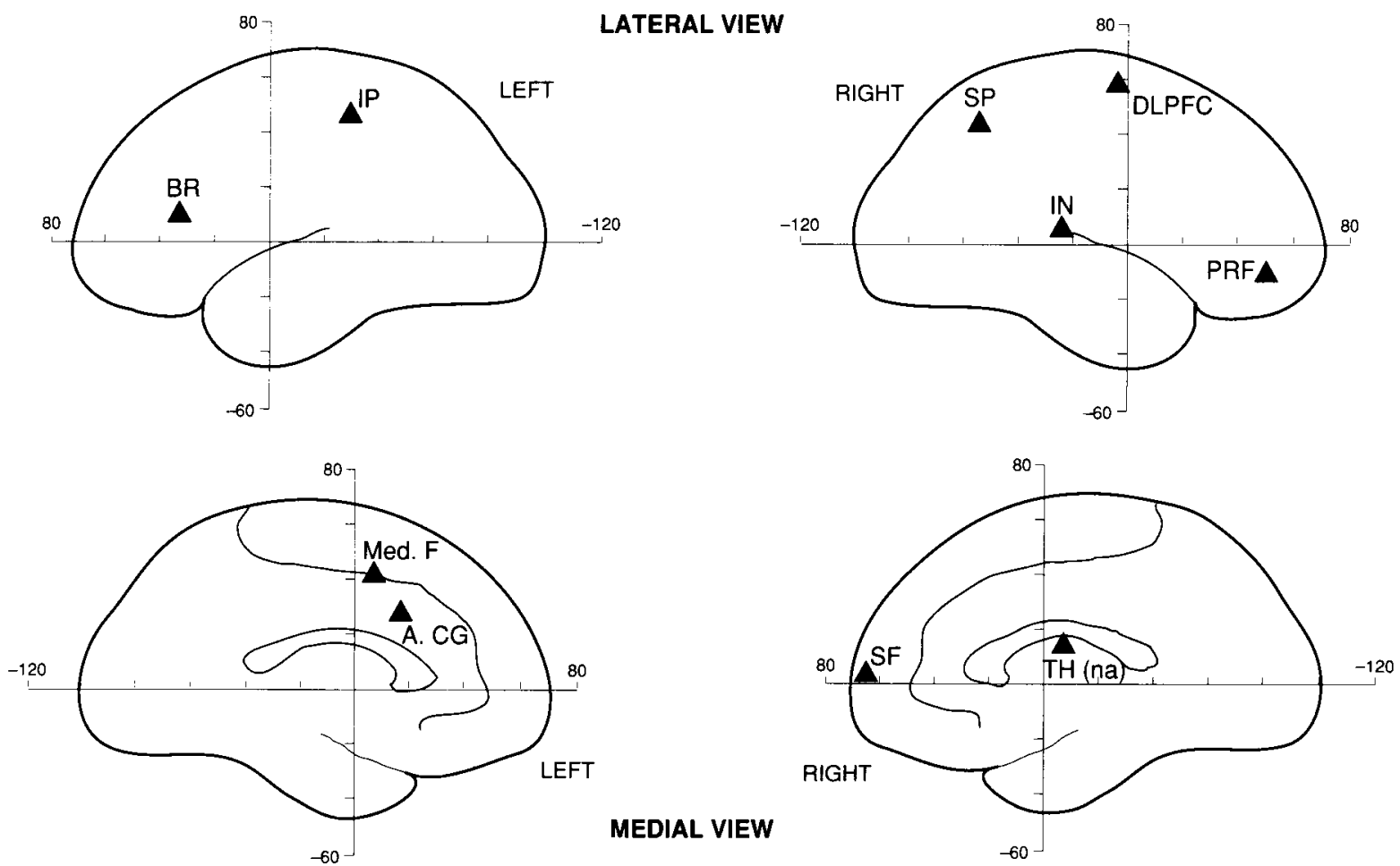

Figure 5. Areas in which there was significantly greater blood flow in the coordinate spatial relations encoding condition than in the baseline condition in Lxperiment 2. Left hemisphere is on the left; right hemisphere is on the right. Points on the lateral surface are at the top; points on the medial surface are at the bottom. Points indicate the location of the most significant pixel in an area. The axes indicate location in $20-\mathrm{mm}$ increments relative to the anterior commissure.

number are in fact within $15 \mathrm{~mm}$ (the smoothness of the image was calculated to be $14.2 \mathrm{~mm}$ ). In the categorical-baseline comparison, right superior parietal cortex was activated at comparable locations in Experiment 1. In the coordinate-baseline comparison, the thalamus was activated in comparable locations. Perhaps most

Table 6

Blood Flow in the Categorical Spatial Relations Encoding Task Compared With the Coordinate Task in Experiment 2, in Which Stimuli Were Presented for 200 msec and Were Aligned Along the Midline

\begin{tabular}{|c|c|c|c|c|c|}
\hline Area & $x$ & $Y$ & $Z$ & $Z$ Score & $\begin{array}{c}p \\
\text { (hyp) }\end{array}$ \\
\hline \multicolumn{6}{|c|}{ Categorical-Coordinate } \\
\hline \multicolumn{6}{|c|}{ Left-Hemisphere Regions } \\
\hline Prefrontal area & -33 & 35 & -4 & 2.87 & .03 \\
\hline \multicolumn{6}{|c|}{ Coordinate-Categorical } \\
\hline \multicolumn{6}{|c|}{ Left-Hemisphere Regions } \\
\hline Inferior parietal & -34 & -57 & 40 & 3.12 & .02 \\
\hline \multicolumn{6}{|c|}{ Right-Hemisphere Regions } \\
\hline Superior parietal & 29 & -63 & 40 & 2.45 & .03 \\
\hline
\end{tabular}

Note-Coordinates are relative to the anterior commissure. $X$ is the horizontal dimension (with positive toward the right); $Y$ is the depth dimension (with positive toward the anterior part of the brain); $Z$ is the vertical dimension (with positive toward the top). The last column of the table indicates the probability $(p)$ levels associated with the listed region, depending on the presence of a hypothesis (hyp) for that region. critically, in the coordinate-categorical comparison, the right inferior parietal lobe, right superior parietal lobe, and right precuneus were activated in comparable locations. No comparable areas were activated in the two experiments for the categorical-coordinate comparison.

\section{GENERAL DISCUSSION}

As expected, we found that our apparently simple tasks draw on large networks of areas and that processes that allocate attention selectively are involved in computing spatial relations. In addition, distinct networks of areas were found to be activated by the two types of spatial relations encoding, which is good evidence that they are in fact carried out by distinct mechanisms. We also found that when we changed the stimuli in relatively minor ways - but kept the tasks identical-the results changed dramatically. Although we found evidence that three right parietal areas are used to compute coordinate spatial relations more than they are used to compute categorical spatial relations, we found no areas that were clearly and consistently used to compute categorical spatial relations more than coordinate spatial relations. However, in the comparisons between the test conditions, it seems that left frontal regions are involved in categorical processing (relative to coordinate processing). This may be of interest because language also has a strong basis in 

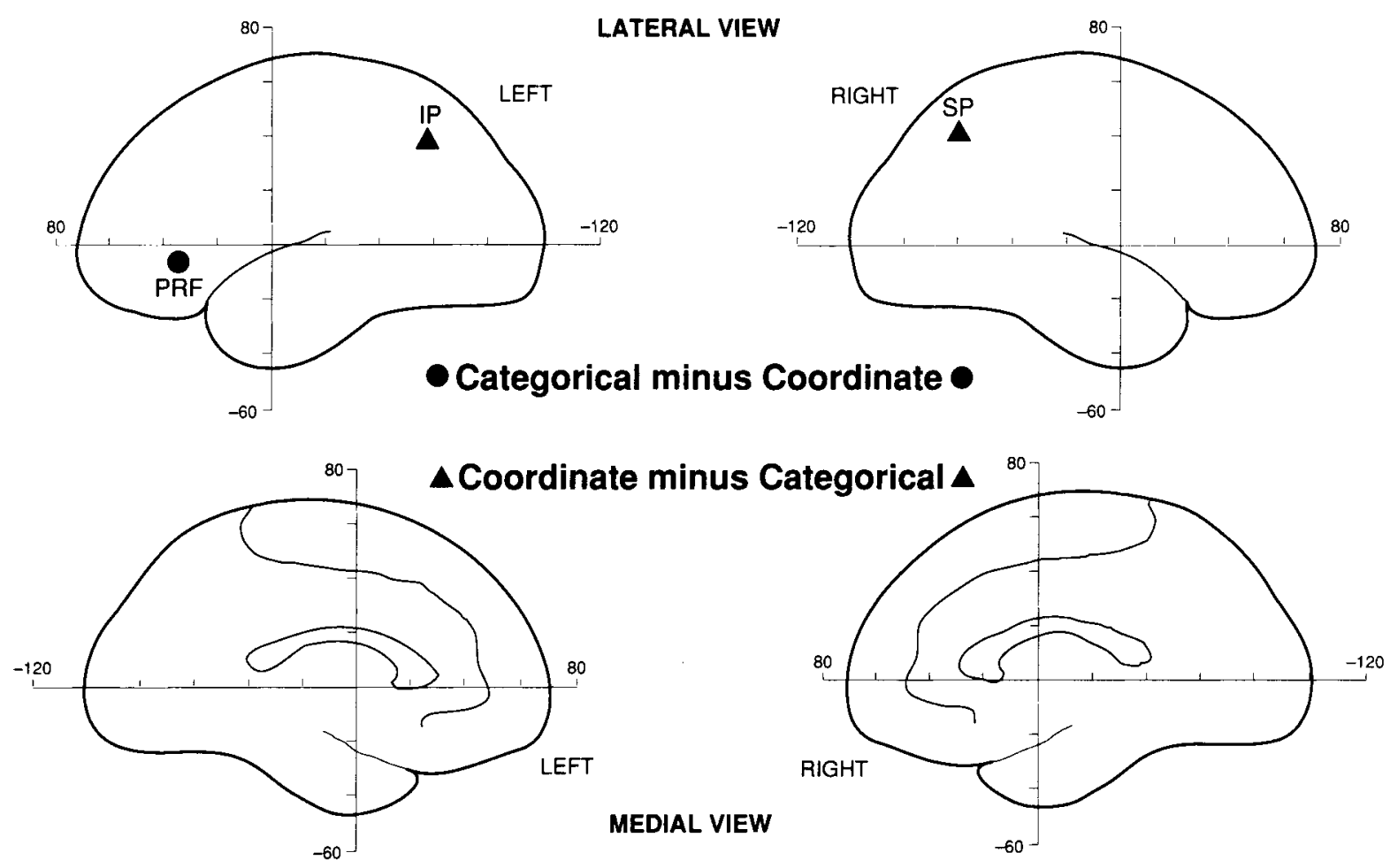

Figure 6. Areas in which there was significantly greater blood flow in the categorical spatial relations encoding condition than in the coordinate spatial relations encoding condition (circles) or vice versa (triangles) in Experiment 2. Left hemisphere is on the left; right hemisphere is on the right. Points on the lateral surface are at the top; points on the medial surface are at the bottom. Points indicate the location of the most significant pixel in an area. The axes indicate location in 20 -mm increments relative to the anterior commissure.

the left frontal cortex and can be conceptualized as a category system. In contrast, parietal regions seem to play a larger role in encoding coordinate spatial relations more than in encoding categorical spatial relations.

We wondered whether our failure to find the predicted differences in inferior parietal activation when we directly compared the test conditions could be ascribed to a simple lack of statistical power. Thus, we lowered the threshold for significance and examined the results. As expected, many more areas were evident in all conditions when we dropped the $Z$ threshold to 1.5 or even 1.0 ; however, we still failed to find evidence of activation in the inferior parietal cortex in the expected locations. Thus, we doubt that power is the crucial factor. Instead, our failure to find the lateralized parietal differences we expected may indicate that the relevant processing is fast and "automatic," and, hence, it does not cause significant changes in regional cerebral blood flow in the situation we examined. Alternatively, it is possible that the parietal lobes do not contribute as much to the encoding of categorical spatial relations as we had assumed. However, Gerstman's (1940) syndrome, which includes difficulty in encoding at least some categorical spatial relations (in particular, left/right), is associated with left inferior parietal damage.
The differences between the results of the two experiments lead us to speculate that although some tasks are accomplished relatively consistently by a single set of brain areas, others may vary across time, even within an individual. The results clearly indicate that a large system, or network, is involved in both the categorical and the coordinate computations. Perhaps when a network is large, a change in even one of the constituent operations will affect the entire system.

Finally, although the two systems clearly are different, the fact that there are relatively few activations that vary between the two experimental conditions (as indicated when they are compared directly with each other) may mean that the systems overlap substantially, and one may even contribute to the other. For example, for some people, some of the time, encoding coordinate spatial relations may involve visualizing an imaginary line where the participant thinks 0.5 in. should be, then making adjustments to the image based on memory. This could involve several above/below decisions before deciding on where the final placement of the line should be. Similarly, locating the bar and $X$ may require encoding coordinates before moving on to the categorical judgment per se. We note, however, that all of the participants claimed to be familiar with the extent of 0.5 in. prior to the study. 
The main purpose of the learning phase was to familiarize them with what the distance looked like on the screen viewed from their perspective. Thus, we doubt that the participants actually had to learn something new, which was kept in mind during the task. Moreover, behaviorally, other tasks that do not rely on this type of judgmentbut, for example, require participants to decide whether two pairs of figures are separated by same extent (e.g., Laeng \& Peters, 1995) - show the same right-hemisphere advantage we observed with this task. Nevertheless, we cannot rule out with certainty a role of memory in our tasks.

In sum, the system used to compute spatial relations representations is more complex than one might expect solely on the basis of a consideration of the tasks themselves, which are deceptively simple. This complexity in turn may afford different processing strategies. Nevertheless, the present results provide clear evidence that there are at least two different ways to encode spatial information, which rely in part on different regions of the brain.

\section{REFERENCES}

ANDERSEN, R.A. (1987). Inferior parietal lobule function in spatial perception and visuomotor integration. In F. Plum \& V. Mountcastle (Eds.), Handbook of physiology: Section I. The nervous system: Vol. 5. Higher functions of the brain (pp. 483-518). Bethesda, MD: American Physiological Society.

BaKer, D. P., Chabris, C. F., \& Kosslyn, S. M. (in press). Encoding categorical and coordinate spatial relations with complete positional uncertainty: New simulation models. Cognitive Science.

BIEDERMAN, I. (1987). Recognition-by-components: A theory of human image understanding. Psychological Review, 94, 115-147.

COOK, N. D., FrüH, H., \& LandIS, T. (1995). The cerebral hemispheres and neural network simulations: Design considerations. Journal of Experimental Psychology: Human Perception \& Performance, 21 , 410-422.

Corbetta, M., Miezen, F. M., Schulman, G. L., \& Petersen, S. E. (1993). A PET study of visuospatial attention. Journal of Neuroscience, 13, 1202-1226.

Delis, D. C., Robertson, L. C., \& Efron, R. (1986). Hemispheric specialization of memory for visual hierarchical stimuli. Neuropsychologia, 24, 205-214.

Friston, K. J., Frith, C. D., Liddle, P. F., \& Frackowiak, R. S. J. (1991). Comparing functional (PET) images: The assessment of significant changes. Journal of Cerebral Blood Flow \& Metabolism, 11 , 690-699.

Fujita, I., Tanaka, K., Ito, M., \& Cheng, K. (1992). Columns for visual features of objects in monkey inferotemporal cortex. Nature, 360, 343-346.

Gerstman, J. (1940). Syndrome of finger agnosia, disorientation for right and left, agraphia and acalculia. Archives of Neurology \& Psychiatr, 44, 398-408.

Goldman-Rakic, P. S. (1987). Circuitry of primate prefrontal cortex and regulation of behavior by representational knowledge. In F. Plum \& V. Mountcastle (Eds.), Handbook of physiology: Secrion I. The nervous system: Vol. 5. Higher functions of the brain (pp. 373-417). Bethesda, MD: American Physiological Society.

Haxby, J. V., Grady, C. L., Horowitz, B., Ungerleider, L. G., Mishioin, M., Carson, R. E., Herscovitch, P., Schapiro, M. B., \& RAPOPORT, S. I. (1991). Dissociation of object and spatial visual processing pathways in human extrastriate cortex. Proceedings of the National Academy of Sciences, 88, 1621-1625.
Hellige, J. B., \& Michimata, C. (1989). Categorization versus distance: Hemispheric differences for processing spatial information. Memory \& Cognition, 17, 770-776.

JACOBS, R. A., \& KossL.YN, S. M. (1994). Encoding shape and spatial relations: The role of receptive field size in coordinating complementary representations. Cognitive Science, 18, 361-386.

Jonides, J., Smith, E. E., Koeppe, R. A., Awh, E., Minoshima, S., \& Mintun, M. A. (1993). Spatial working memory in humans as revealed by PET. Nature, 363, 623-625.

KoENig, O., Reiss, L. P., \& Kosslyn, S. M. (1990). The development of spatial relation representations: Evidence from studies of cerebral lateralization. Journal of Experimental Child Psychology, 50, 119-130.

KOLB, B., \& WhISHAW, I. Q. (1990). Fundamentals of human neuropsychology (3rd ed.). New York: W. H. Freeman.

KossLyN, S. M. (1987). Seeing and imagining in the cerebral hemispheres: A computational approach. Psychological Review, 94, 148175.

KoSSLYN, S. M. (1994). Image and brain: The resolution of the imagery debate. Cambridge, MA: MIT Press.

Kosslyn, S. M., Alpert, N. M., Thompson, W. L., Chabris, C. F., RAUCH, S. L., \& ANDERSON, A. K. (1994). Identifying objects seen from different viewpoints: A PET investigation. Brain, 117, 10551071 .

Kosslyn, S. M., Anderson, A. K., Hilger, L. A., \& Hamilton, S. E. (1994). Hemispheric differences in sizes of receptive fields or attentional biases? Neuropsychology, 8, 139-147.

Kosslyn, S. M., Chabris, C. F., Marsolek, C. J., \& Koenig, O. (1992). Categorical versus coordinate spatial relations: Computational analyses and computer simulations. Journal of Experimental Psychology: Human Perception \& Performance, 18, 562-577.

Kosslyn, S. M., Chabris, C. F., Marsolek, C. J., \& KoeniG, O. (1995). On computational evidence for different types of spatial relations encoding: Reply to Cook et al. Journal of Experimental Psychology: Human Perception \& Performance, 21, 423-431.

Kosslyn, S. M., \& KoenIG, O. (1995). Wet mind: The new cognitive neuroscience. New York: Free Press.

Kosslyn, S. M., Koenig, O., Barrett, A., Cave, C. B., Tang, J., \& Gabrieli, J. D. E. (1989). Evidence for two types of spatial representations: Hemispheric specialization for categorical and coordinate relations. Journal of Experimental Psychology: Human Perception \& Performance, 15, 723-735.

LAENG, B. (1994). Lateralization of categorical and coordinate spatial functions: A study of unilateral stroke patients. Journal of Cognitive Neuroscience, 6, 189-203.

LAENG, B., \& Peters, M. (1995). Cerebral lateralization for the processing of spatial coordinates and categories in left- and righthanders. Neuropsychologia, 33, 421-439.

LEVINE, D. N. ( 1982). Visual agnosia in monkey and man. In D. J. Ingle, M. A. Goodale, \& R. J. W. Mansfield (Eds.), Analysis of visual behavior (pp. 629-670). Cambridge, MA: MIT Press.

MARR, D. (1982). Vision: A computational investigation into the human representation and processing of visual information. New York: W. H. Freeman.

MESUlam, M.-M. (1981). A cortical network for directed attention and unilateral neglect. Annals of Neurology, 10, 309-325.

O'Reilly, R. C., Kosslyn, S. M., Marsolek, C. J., \& Chabris, C. J. (1990). Receptive field characteristics that allow parietal lobe neurons to encode spatial properties of visual input: A computational analysis. Journal of Cognitive Neuroscience, 2, 141-155.

PAUS, T. (1996). Location and function of the human frontal eye-field: A selective review. Neuropsvichologia, 34, 475-483.

Posner, M. I., \& Petersen, S. E. (1990). The attention system of the human brain. Annual Review of Neuroscience, 13, 25-42.

Robertson, L. C., \& Delis, D. C. (1986). "Part-whole" processing in unilateral brain damaged patients: Dysfunction of hierarchical organization. Neuropsychologia. 24. 363-370.

SERGENT, J. (1982). The cerebral balance of power: Confrontation or cooperation? Journal of Experimental Psychology: Human Percepfion \& Performance, 8, 253-272. 
Sergent, J., Ohta, S., \& MacDonald, B. (1992). Functional neuroanatomy of face and object processing: A positron emission tomography study. Brain, 115, 15-36.

Sergent, J., Zuck, E., Lévesque, M., \& MacDonald, B. (1992). Positron emission tomography study of letter and object processing: Empirical findings and methodological considerations. Cerebral Cortex, 2, 68-80.

Smith, E. E., \& Jonides, J. (1994). Working memory in humans: Neuropsychological evidence. In M. S. Gazzaniga (Ed.), The cognitive neurosciences (pp. 1009-1020). Cambridge, MA: MIT Press.

Talairach, J., \& Tournoux, P. (1988). Co-planar stereotaxic atlas of the human brain (M. Rayport, Trans.). New York: Thieme.

UNGERLEIDER, L. G., \& HAXBY, J. V. (1994). "What" and "where" in the human brain. Current Opinion in Neurobiology, 4, 157-165.

UNGerLeIDER, L. G., \& MishKin, M. (1982). Two cortical visual systems. In D. J. Ingle, M. A. Goodale, \& R. J. W. Mansfield (Eds.), Analysis of visual behavior (pp. 549-586). Cambridge, MA: MIT Press.

\section{NOTES}

1. The left-hemisphere advantage for categorical relations encoding is rarely significant in a single experiment, but it is virtually always present; this consistent trend reaches statistical significance in a metaanalysis (e.g., see Kosslyn, Chabris, Marsolek, \& Koenig, 1992).

2. Most neurons in the inferior temporal (IT) cortex respond most strongly to stimuli on the fovea, whereas most posterior parietal (PP) neurons respond weakly to stimuli on the fovea (this is "foveal sparing"; for a review, see O'Reilly, Kosslyn, Marsolek, \& Chabris, 1990). $O$ 'Reilly et al. have reported computer simulations that showed that neural networks could more effectively compute location when the input units were hard-wired with receptive fields like those in PP than when they were hard-wired with receptive fields like those in IT. Thus, size per se is not the only crucial characteristic.

(Manuscript received December 4, 1997; revision accepted for publication June 1, 1998.) 